\title{
Consumo de Energia em Função da Taxa de Transmissão e do Tamanho do Pacote em Redes Ad Hoc Aloha de um Salto
}

\author{
Bruna A. da Silva ${ }^{1}$, Renato M. de Moraes ${ }^{1}$ \\ ${ }^{1}$ Centro de Informática - Universidade Federal de Pernambuco (UFPE) \\ CEP 50740-560 - Recife - PE - Brasil \\ bas4@cin.ufpe.br, renatomdm@cin.ufpe.br
}

\begin{abstract}
The autonomy of a communication device, together with its form of operation, is related to its energy consumption. In ad hoc and sensor wireless networks we seek that the device can operate with high efficiency and with minimum human interference as possible during its operation. In this paper, a study was carried out on the energy expended to perform the communication at one hop $E_{1 \text { hop }}$ (energy consumption per bit for one-hop successful transmission) employing slotted Aloha medium access control protocol in order to provide, at the physical and link layer level, the optimization of the transmission power $\left(P_{t}\right)$ as a function of packet length $\left(N_{b}\right)$ and data transmission rate $(R)$. Our results indicate that there are possible optimum values of operations for $P_{t}$ as a function of $N_{b}$ and $R$ which result in reduced energy consumption for such networks.
\end{abstract}

Resumo. O aumento da autonomia de um dispositivo de comunicação, aliado à sua forma de operação, está relacionado com seu consumo de energia. Em redes ad hoc e de sensores sem fio deseja-se que o dispositivo possa operar com a melhor eficiência possível e com o mínimo de interferência humana durante seu funcionamento. Neste artigo, um estudo foi realizado sobre a energia gasta para realização da comunicação em um salto $E_{1 h o p}$ (energia consumida por bit para transmissão com sucesso em um salto) empregando slotted Aloha como protocolo de acesso ao meio a fim de proporcionar, na camada física e de enlace, a otimização da potência de transmissão $\left(P_{t}\right)$ em função do número de bits do pacote $\left(N_{b}\right)$ e da taxa de transmissão de dados $(R)$. Nossos resultados indicam que é possivel se obter valores ótimos de $P_{t}$ em função de $N_{b}$ e $R$ que resultam em reduzido consumo de energia nestas redes.

\section{Introdução}

A causa predominante do consumo de energia em redes sem fio ad hoc e também de sensores está relacionada à comunicação. Esse consumo de energia, contudo, é em geral diretamente relacionado a diversos parâmetros das camadas física e de enlace. Por outro lado, deseja-se que os dispositivos destas redes consigam se autoajustar às diversas condições de operações de forma a resultar no melhor desempenho dentre as diversas métricas de funcionamento. Em redes ad hoc e de sensores que operam com bateria, o consumo de energia é uma das principais medidas de desempenho, pois está diretamente ligada à duração (ou longevidade) de operação dos dispositivos que a compõem [Gupta e Shekokar 2016], [Mobin et al. 2017].

Em [Gupta e Shekokar 2016] um novo modelo é proposto a fim de promover o tempo de vida de um nó sensor por otimização do tamanho do pacote, de modo que 
não haja perdas. Considerando que o consumo de energia é diretamente proporcional ao tamanho do pacote e a distância entre os vários nós participantes na comunicação, os autores propuseram um algoritmo que consiste de quatro fases. Nesse contexto, o tempo de vida da rede é usado como um parâmetro para analisar e mostrar que a rede pode ser energeticamente eficiente. Porém, esse trabalho focou apenas no tamanho do pacote.

Para [Abdulhadi et al. 2013] a eficiência energética é usada como uma métrica de otimização cujo problema é formulado como não linear, ou seja, cuja definição consiste em um sistema de equações e desigualdades, coletivamente denominadas restrições, por meio de um conjunto desconhecido de variáveis reais que, juntamente a uma função objetivo, podem ser maximizadas ou minimizadas. Em seu trabalho o autor formula a eficiência energética e o problema da otimização do pacote, buscando um tamanho de pacote ótimo que maximiza a eficiência energética em redes ad hoc sem fio de comunicação cooperativa. Novamente o foco do estudo foi o tamanho do pacote.

Diante do estudo desenvolvido por [Akbas et al. 2016], mostrou-se que há um tamanho de pacote ótimo para cada cenário especificado onde o tempo de vida da rede é maior. No entanto, este resultado é observado apenas se o nível de sensibilidade do receptor é baixo a ponto de permitir que transceptores sejam capazes de operar em uma parcela significativa da região de transição tal como especificado em [Zuniga e Krishnamachari 2004]. O principal objetivo do trabalho foi caracterizar o impacto do tamanho do pacote e dos níveis da potência de transmissão no que diz repeito ao tempo de vida da rede.

Assim, motivados pela necessidade de explorar novas abordagens que considerem não apenas o tamanho do pacote, mas também outros importantes parâmetros como a taxa de transmissão e a potência de transmissão, que podem ser automaticamente ajustáveis pelos próprios dispositivos, o presente trabalho busca analisar o consumo de energia para a realização da comunicação a um salto em função desses parâmetros, considerando também o protocolo de acesso ao meio. Aqui, para simplificar a análise, todos os nós estão ao alcance dos demais, não sendo portanto considerado o efeito do terminal oculto, caracterizando assim uma rede de um único salto.

Este trabalho está dividido conforme descrito a seguir. A Seção 2 aborda os trabalhos relacionados. A Seção 3 revisita a formulação de [Zhang e Gorce 2008] e em seguida a ampliamos para incluir o efeito do protocolo de comunicação Aloha e agora com foco no consumo de energia em função da taxa de transmissão de dados $(R)$, do tamanho do pacote $\left(N_{b}\right)$ para uma potência ótima de transmissão. Os resultados e discussão são apresentados na Seção 4 e a Seção 5 conclui o artigo.

\section{Trabalhos Relacionados}

Tendo em vista que geralmente a distribuição dos nós em redes ad hoc e de sensores são nos mais variados ambientes e que lá permanecerão por tempo indeterminado, sem que ocorra recarga de suas baterias, é de fundamental importância que possuam um consumo de energia mínimo a fim de que se conservem ativos pelo máximo de tempo possível. Uma vez que esses nós sejam utilizados, por exemplo, para aplicações de monitoramento e sensoriamento, é primordial a realização de estudos do consumo de energia de cada um dos componentes dessa rede [Rana e Zaveri 2011], [Stankovic e He 2012], [Ye et al. 2002], [Feeney e Nilsson 2001]. 
Por se tratar de redes que apresentam seu desempenho relacionado com o consumo de energia, contenção e colisão de pacotes, o tema abordado neste trabalho vem sendo amplamente discutido. Em [Tang et al. 2017] um estudo é desenvolvido com base em métodos de redução do consumo de energia de protocolos de roteamento geográficos. Esses tipos de protocolo roteiam um pacote salto-a-salto até que se chegue ao destino. Neste contexto, o nó transmissor precisa obter a informação de sua posição geográfica, da posição de seus vizinhos de um salto e do nó receptor. Destaca, ainda, o consumo de energia na transmissão de um pacote de dados, a um salto, do nó transmissor para o nó receptor, incluindo as retransmissões. Contudo, o trabalho não considera a taxa de erro de bit (BER, do inglês Bit Error Rate) e nem a energia gasta para enviar um bit com sucesso. Também não faz análises referentes ao tamanho do pacote de dados e nem à taxa de transmissão.

[Ito et al. 2016], por sua vez, desenvolve um protocolo para redes ad hoc utilizando BLE (do inglês Bluetooth Low Energy) e realiza seu experimento considerando a latência medida em um salto. Porém, também não é verificado em suas análises um estudo sobre a taxa de erro de bit, sobre a energia gasta para a realização da transmissão de um pacote de bits a um salto e nem sobre o efeito do tamanho do pacote nessa transmissão.

Um algoritmo genético é utilizado em [Shehab et al. 2017] para maximizar a vazão e minimizar o consumo de energia em redes, cujo os nós de um mesmo cluster estão a um único salto um do outro. Considera como parâmetros a distância média entre os nós e o consumo esperado de energia. No entanto, não é feito um estudo sobre a influência de protocolos, a nível de camada de enlace, que certifiquem a probabilidade de um dado nó transmitir com sucesso considerando o número de retransmissões necessárias em decorrência das eventuais colisões.

Em [Sadiq et al. 2017] é feito um estudo na qual a camada de rede utiliza as informações da camada física e da camada de enlace a fim de encontrar um caminho para o envio de dados que promova uma comunicação energeticamente eficiente. Foram considerados parâmetros como o número de nós, a distância entre os nós, a taxa de erro de bit, o consumo de energia de cada nó, a potência de transmissão, dentre outros. É empregada uma análise em termos da taxa de transmissão de dados e do consumo de energia por bit. No entanto, não se faz um estudo detalhado em termos do tamanho do pacote, bem como da energia gasta para a realização em cada salto.

Um esquema de otimização adaptativa do consumo dinâmico de energia foi proposto por [Liu et al. 2015]. Nesse contexto, a otimização do consumo de energia é feito em função do BER, da distância de referência em transmissão de sinais sem fio, do número de nós em um cluster, dos pacotes codificados na rede e do consumo de energia para enviar ou receber um bit. Por outro lado, não é explorada a probabilidade de sucesso na entrega do dado, não faz uso de nenhum protocolo de camada de enlace e nem verifica a influência da taxa de transmissão no consumo de energia da rede.

[Goratti et al. 2014], em seu artigo, faz uso do protocolo slotted Aloha a fim de obter um sistema livre de colisão, uma vez que nesse tipo de protocolo, um nó transmite por vez. Em sua abordagem, considera o consumo de energia e toma como parâmetros a potência e a taxa de transmissão. No entanto, não considera parâmetros como o tamanho do pacote e a taxa de erro de bit. [Li et al. 2015], por sua vez, faz uso do protocolo slotted 
Aloha como objeto de comparação em redes de sensores. Faz análises do consumo de energia apenas baseadas na densidade da rede e não considera a influência de parâmetros como a taxa de transmissão e o tamanho do pacote.

Em [Gao 2002], [Gorce et al. 2007] e [Zhang e Gorce 2008] foi desenvolvido um robusto modelo de consumo de energia para redes ad hoc e de sensores onde foi levado em consideração diversos parâmetros reais da camada física. Denominada EDRb (do inglês Energy Distance Ratio per bit), cuja unidade é dada em Joule por bit por metro (J/bit/m), essa métrica indica o consumo energético em Joules para se enviar um bit de dado sobre uma distância padrão de um metro. Deste modo, quanto menor for o valor de EDRb, mais eficiente energeticamente a rede se comporta e, portanto, menor o consumo de energia resulta.

A eficiência energética expressa nessa métrica traz consigo o embasamento necessário para estudo e análise de parâmetros como o raio de transmissão ótimo, cuja definição representa o alcance ideal no qual um nó possa enviar informação e esta ser recebida com êxito. Caso o comprimento do salto de comunicação entre os nós seja muito grande, será necessário gastar uma maior quantidade de energia para viabilizar a comunicação de forma a não ocorrer falha na comunicação e consequente redução da conectividade na rede. Por outro lado, se o comprimento do salto for muito pequeno, a energia gasta em cada salto será bem menor, mas se fará necessário mais saltos para se alcançar o destino resultando em um consumo acumulado demasiado.

O estudo detalhado do consumo de energia apresentado por [Zhang e Gorce 2008] procurou determinar a distância ótima de comunicação entre os nós em função da potência de transmissão. Entretanto, em casos práticos, o posicionamento dos nós pode não ser um parâmetro controlado com muita precisão, seja porque os nós são dispostos na rede de forma aleatória, ou porque a possibilidade de mobilidade aleatória deva ser levada em consideração. Também não foi levado em consideração o efeito do protocolo de acesso ao meio.

No presente artigo, portanto, é realizado um estudo do consumo de energia da comunicação em um salto. Diferentemente das análises citadas anteriormente, trazemos agora uma investigação do efeito conjunto da comunicação a um salto em função da taxa de transmissão de dados $(R)$ e do tamanho do pacote $\left(N_{b}\right)$ empregando a potência de transmissão $\left(P_{t}\right)$ ótima levando também em consideração o protocolo de acesso ao meio (MAC, do inglês medium access control). Consideramos a possibilidade da variação da distância entre os nós comunicantes e a probabilidade de sucesso do nó transmitir através do uso do protocolo MAC slotted Aloha [Roberts 1975] que foi escolhido para análise devido a sua simplicidade de modelagem. Entretanto, a metodologia aqui empregada pode ser estendida para outros protocolos de acesso ao meio.

\section{Consumo de Energia}

Utilizamos como ponto de partida a formulação da energia consumida para a realização de um salto com retransmissão desenvolvida em [Zhang e Gorce 2008] que atualizou a descrição de [Karl e Willig 2007] e empregou as propostas de [Gao 2002] e [Gorce et al. 2007]. Trata-se, portanto, de um modelo robusto e optamos partir deste por verificarmos que o mesmo está bem definido, incorpora vários outros parâmetros de camada física e permitiu sua alteração para considerar o protocolo MAC. 
Nossa análise considera uma rede sem fio contendo $n$ nós e parte do pressuposto que todos os nós estão ao alcance uns dos outros, caracterizando uma rede $a d$ hoc sem fio de um único salto. Isso permite simplificar a análise, pois não será considerado o efeito do terminal oculto. Entretanto, um dos nossos trabalhos futuros será estender a análise para uma rede com múltiplos saltos.

O consumo de energia $\left(E_{p}\right)$ para o envio de um pacote é a soma entre a energia empregada na transmissão $E_{T x}$ e a energia gasta para recepção $E_{R x}$. Assim, resulta que [Zhang e Gorce 2008]

$$
E_{p}=E_{T x}+E_{R x}
$$

$E_{T x}$ e $E_{R x}$ são descritas, respectivamente, de acordo com [Karl e Willig 2007] por

$$
E_{T x}=T_{\text {start }} P_{\text {start }}+\frac{N_{b}}{R}\left(P_{\text {txElec }}+P_{\text {amp }}\right)
$$

$\mathrm{e}$

$$
E_{R x}=T_{\text {start }} P_{\text {start }}+\frac{N_{b}}{R} P_{\text {rxElec }},
$$

onde $N_{b}$ é o número de bits por pacote, $R$ é a taxa de transmissão de dados, $P_{t x E l e c}$ é a potência para alimentação do circuito de transmissão, $P_{r x E l e c}$ é a potência para alimentação do circuito de recepção, $T_{\text {start }}$ é o tempo de inicialização do circuito e $P_{\text {start }}$ é a potência requerida para inicialização. $P_{a m p}$ é definido como a potência para amplificação do sinal na transmissão, e é dada por

$$
P_{a m p}=\alpha_{a m p}+\beta_{a m p} P_{t},
$$

em que $\alpha_{a m p}$ é um nível constante de potência e $\beta_{a m p}$ é uma constante de proporcionalidade da potência de transmissão $P_{t}$.

A fim de obter o consumo médio de energia por bit para uma transmissão a um salto com sucesso, deve-se dividir o consumo para transmissão de um pacote pelo número de bits $N_{b}$ deste pacote. Consequentemente, define-se o consumo de energia por bit $E_{b}$ (expresso em Joules por bit $(J / b i t))$ como sendo

$$
E_{b}=\frac{E_{p}}{N_{b}}=E_{c}+K_{1} P_{t}
$$

onde $E_{c}$ e $K_{1}$ são dados, respectivamente, por

$$
\begin{gathered}
E_{c}=\frac{2 T_{\text {start }} P_{\text {start }}}{N_{b}}+\frac{P_{\text {txElec }}+P_{r x E l e c}+\alpha_{a m p}}{R}, \\
K_{1}=\frac{\beta_{a m p}}{R} .
\end{gathered}
$$

Por outro lado, para um canal não-confiável, a probabilidade de sucesso de transmissão de um pacote contendo $N_{b}$ bits é $p_{l}(\gamma)=(1-B E R(\gamma))^{N_{b}}$ em que $B E R(\gamma)$ é a taxa de erro de bits em função da relação sinal-ruído $(\gamma)$ [Gorce et al. 2007]. Aqui modificamos essa probabilidade para incluir o efeito do protocolo slotted Aloha, em que a probabilidade de acesso ao meio com sucesso de um nó é dada por $\delta(n)=\frac{1}{n}\left(1-\frac{1}{n}\right)^{n-2}$ 
para $n$ nós na rede. Logo, resulta que a probabilidade de transmissão com sucesso de um pacote é

$$
P_{s}=p_{l}(\gamma) \delta(n)=\frac{(1-B E R(\gamma))^{N_{b}}\left(\frac{n-1}{n}\right)^{n-2}}{n} .
$$

A relação sinal-ruído é definida como sendo

$$
\gamma=C_{2} P_{t} d^{-\alpha} R^{-1}
$$

em que $d$ indica a distância entre dois nós comunicantes, sendo $\alpha$ o coeficiente de atenuação do sinal com a distância, e $P_{t}$ a potência de transmissão. A constante $C_{2}$ é expressa por

$$
C_{2}=\frac{G_{T} G_{R} \lambda^{2}}{(4 \pi)^{2} N_{0}}
$$

onde $G_{T}$ e $G_{R}$ representam, respectivamente, os ganhos das antenas no transmissor e no receptor, $N_{0}$ é a densidade espectral de potência de ruído no canal de comunicação, $\lambda$ é o comprimento de onda do sinal transmitido e igual a $\frac{c}{f_{c}}$ em que $c$ é a velocidade da luz e $f_{c}$ é a frequência da onda portadora.

É importante notar que a relação sinal-ruído não deve considerar a interferência causada pelos demais nós da rede visto que isto é tratado no protocolo de controle de acesso ao meio. Assim, se houver transmissão simultânea de dois ou mais nós, os pacotes de dados colidem entre si e a informação é perdida ocasionando necessariamente a retransmissão, sendo tal efeito incorporado aqui no protocolo Aloha.

A partir de então, podemos definir o consumo médio de energia por bit para um único salto, em unidades de Joules/bit, como

$$
\bar{E}_{1 h o p}=E_{b}\left(P_{t}\right) \sum_{k=1}^{\infty} k P_{s}\left(1-P_{s}\right)^{k-1}=\frac{E_{b}\left(P_{t}\right)}{P_{s}}
$$

onde $k$ é o número de retransmissões necessárias para o envio do pacote de dados com sucesso. Sendo assim, o consumo médio de energia por bit para uma transmissão bem sucedida a um salto resulta

$$
\bar{E}_{1 h o p}=\frac{\left(E_{c}+K_{1} P_{t}\right)}{(1-B E R(\gamma))^{N b}} \cdot \mu
$$

em que

$$
\mu=\frac{n}{\left(\frac{n-1}{n}\right)^{n-2}}
$$

O canal de comunicação que este trabalho considera é o que emprega o desvanecimento Rayleigh, que é amplamente utilizado na literatura em casos de redes ad hoc e de sensores para modelar canais sem fio e sem linha de visada direta entre as antenas comunicantes. Para uma relação sinal-ruído maior que 5, que é o caso para os valores de parâmetros aqui empregados, pode-se aproximar taxa de erro de bit por [Goldsmith 2005]

$$
\operatorname{BER}(\gamma) \approx \frac{\alpha_{m}}{2 \beta_{m} \gamma}
$$


onde $\alpha_{m}$ e $\beta_{m}$ são constantes que dependem da modulação empregada. Será considerada a modulação BPSK (do inglês Binary Phase Shift Keying), que é comumente encontrada nos dispositivos de comunicação sem fio, e nesse caso $\alpha_{m}=1$ e $\beta_{m}=2$. Substituindo a Eq. (14) em (12), resulta que

$$
\bar{E}_{1 h o p}=\frac{E_{c}+K_{1} P_{t}}{\left(1-\frac{\alpha_{m}}{2 \beta_{m} C_{2} P_{t} d^{-\alpha} R^{-1}}\right)^{N_{b}}} \cdot \mu .
$$

É possível, ainda, determinar a potência de transmissão ótima $\left(P_{0}\right)$, para posterior ajuste conforme as variações da taxa de transmissão $(R)$ e do tamanho do pacote $\left(N_{b}\right)$, através da resolução da derivada parcial a seguir

$$
\left.\frac{\partial \bar{E}_{1 h o p}}{\partial P_{t}}\right|_{P_{t}=P_{0}}=0
$$

Desenvolvendo essa equação, obtém-se a potência de transmissão ótima em função da taxa de transmissão $(R)$ e em função do tamanho do pacote $\left(N_{b}\right)$, ou seja,

$$
P_{0}(R, N b)=\frac{d^{\alpha} \alpha_{m}\left(1+N_{b}\right)}{4 C_{2} \beta_{m} R^{-1}}+\frac{\sqrt{d^{\alpha} \beta_{a m p} \alpha_{m}\left(d^{\alpha} \beta_{a m p} \alpha_{m}\left(1+N_{b}\right)^{2}+8 \beta_{m} C_{2} R^{-1}\left(C_{3} R+C_{4} N b\right)\right)}}{4 C_{2} \beta_{a m p} \beta_{m} R^{-1}} .
$$

As constantes $C_{3}, C_{4}$ são oriundas do desmembramento de $E_{c}$, para representação em função de $R$ e $N_{b}$, e são dadas, respectivamente, por

$$
\begin{gathered}
C_{3}=2 T_{\text {start }} P_{\text {start }}, \\
C_{4}=P_{\text {txelec }}+P_{\text {rxelec }}+\alpha_{\text {amp }} .
\end{gathered}
$$

Diante das equações acima apresentadas se propõe, portanto, avaliar o consumo médio de energia por bit para transmissão bem sucedida em um salto em função da taxa de transmissão de dados $(R)$ e do tamanho do pacote $\left(N_{b}\right)$ empregando-se a potência ótima de transmissão em função destes parâmetros (isto é $P_{t}=P_{0}\left(R, N_{b}\right)$ ). Assim, $R$ e $N_{b}$ podem ser variados a fim de atingir valores ótimos de desempenho fixando os demais parâmetros de projeto que estão descritos na Tabela 1. Desta forma, das Eqs. (15) e (17), tem-se que $\bar{E}_{1 h o p}$ pode ser dado em função de $R$ e de $N_{b}$ para os valores ótimos de $P_{0}(R, N b)$, da seguinte forma

$$
\bar{E}_{1 h o p}\left(P_{t}=P_{0}(R, N b)\right)=\frac{C_{3} R+\left(C_{4}+\beta_{a m p} P_{0}\left(R, N_{b}\right)\right) N_{b}}{R N_{b}\left(1-\frac{\alpha_{m}}{2 \beta_{m} C_{2} P_{0}\left(R, N_{b}\right) d^{-\alpha} R^{-1}}\right)^{N_{b}}} \cdot \mu,
$$

onde $P_{0}\left(R, N_{b}\right)$ é dado pela Eq. (17).

\section{Resultados e Discussões}

No intuito de explorar o desempenho energético das redes ad hoc e de sensores aqui modeladas, realizou-se simulações numéricas para transmissão de um salto e apresentamos os resultados para as Eqs. (17) e (20). 


\section{Tabela 1. Parâmetros empregados no modelo de consumo de energia [Karl e Willig 2007], [Zhang e Gorce 2008]}

\begin{tabular}{|c|c|c|}
\hline Variável & Descrição & Valor \\
\hline$P_{r x E l e c}$ & Potência para recepção & $279 \mathrm{~mW}$ \\
$P_{\text {start }}$ & Potência para inicialização & $58,7 \mathrm{~mW}$ \\
$T_{\text {start }}$ & Tempo de inicialização & $446 \mu \mathrm{s}$ \\
$P_{t x E l e c}$ & Potência para transmissão & $151 \mathrm{~mW}$ \\
$n$ & Número de nós & 2 ou 10 \\
$\alpha_{a m p}$ & Nível de potência Eq. (4) & $174 \mathrm{~mW}$ \\
$\beta_{\text {amp }}$ & Constante de proporcionalidade Eq. (4) & 5 \\
$N_{0}$ & Densidade espetral de ruído & $-154 \mathrm{dBm} / \mathrm{Hz}$ \\
$f_{c}$ & Frequência da portadora & $2,4 \mathrm{GHz}$ \\
$c$ & Velocidade da luz & $3 \times 10^{8} \mathrm{~m} / \mathrm{s}$ \\
$G_{T}$ & Ganho da antena na transmissão & 1 \\
$G_{R}$ & Ganho da antena na recepção & 1 \\
$\alpha$ & Nível de atenuação de percurso & 3 \\
$d$ & Distância entre o par de nós comunicantes & $10 \mathrm{~m}, 30 \mathrm{~m}, 50 \mathrm{~m}$ \\
\hline
\end{tabular}

Buscou-se, portanto, dentre as variações no tamanho do pacote e na taxa de transmissão de dados, os valores ótimos dessas variáveis que retornassem o menor consumo de energia por bit para um salto e, consequentemente, maior eficiência energética do sistema. Os valores dos parâmetros empregados estão descritos na Tabela 1, que representam as características físicas do transceptor $\mu$ AMPS-1 [Karl e Willig 2007].

As Figuras 1 e 2 mostram o desempenho da potência de transmissão ótima (Eq. (17)), em Watts (W), em função da taxa de transmissão $R$ e do tamanho do pacote $N_{b}$, respectivamente. Em ambos os casos observa-se que a potência ótima necessária para envio de um pacote aumenta com a taxa de transmissão, com o tamanho do pacote e também com a distância entre o par de nós comunicantes.

Nas Figuras 3 e 5 é possível verificar o consumo médio de energia por bit (em unidades de $\mathrm{dBmJ} / \mathrm{bit}$ ) para uma transmissão bem sucedida em um salto (Eq. (20)). A análise de ambas figuras difere na quantidade de nós que constitui a rede em questão.

Na Figura 3 observa-se que a energia necessária para transmitir um bit diminui com o aumento da taxa de transmissão e com a redução da distância entre os nós. Porém, transmitir a altas taxas demandará um aumento na potência de transmissão ótima requerida. Deve-se, portanto, respeitar-se o limite da taxa de transmissão em que não resulte uma potência acima do permitido pelo hardware do nó transmissor.

Outro aspecto importante de projeto é se empregar uma taxa de transmissão que resulte uma taxa de erros de bit aceitável, pois a BER é uma função direta de $R$ conforme Eqs. (9) e (14). A Figura 4 ilustra o comportamento da taxa de erros de bit em função da taxa de transmissão de dados e percebe-se que o desempenho do sistema nesse quesito piora com o aumento de $R$. Portanto, deve haver um compromisso no uso deste parâmetro. 


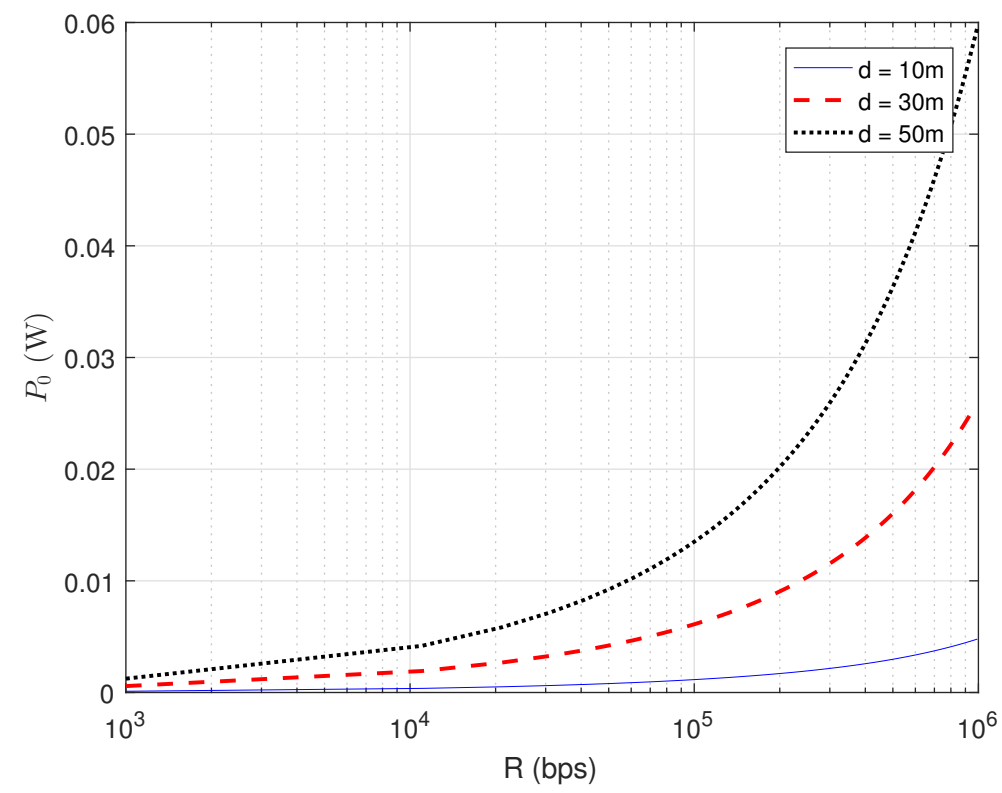

Figura 1. Potência ótima de transmissão em função da taxa de transmissão de dados $(R)$ com o tamanho do pacote $N_{b}=100$ bits e $n=10$ nós.

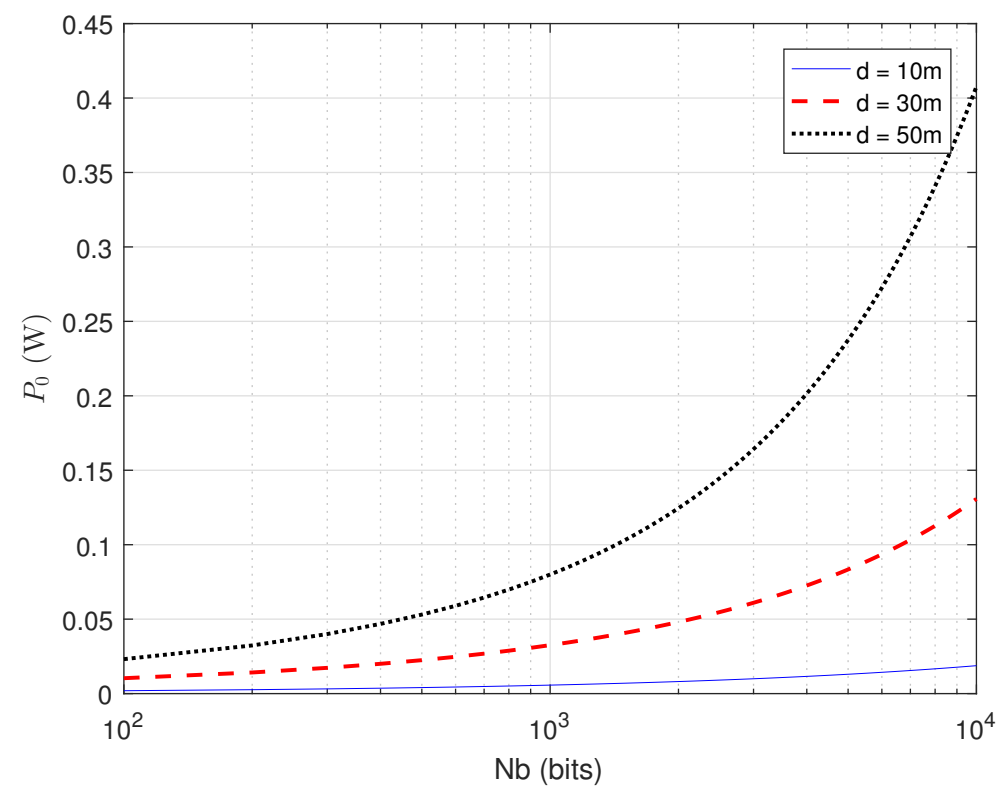

Figura 2. Potência ótima de transmissão em função do tamanho do pacote $\left(N_{b}\right)$ com uma taxa de transmissão $R=250$ kbps e $n=10$ nós.

Para efeito de comparação, a Figura 5 mostra o consumo de energia, quando não existem nós interferentes, ou seja, no cenário em que há apenas dois nós se comunicando $(n=2)$ onde um desses nós transmite e o outro recebe. Como esperado, devido a ausência de nós interferentes na rede, observa-se um menor consumo de energia em função da taxa de transmissão quando se compara com a Figura 3.

As Figuras 6 e 7 retratam o consumo médio de energia por bit para um salto em 


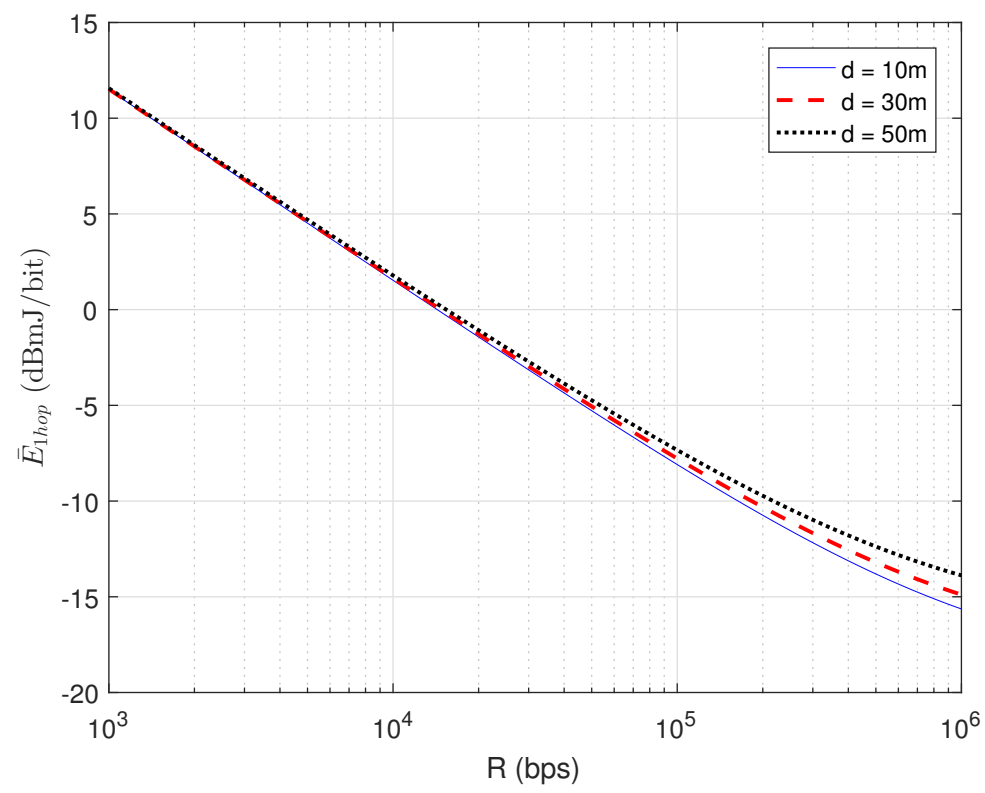

Figura 3. $\bar{E}_{1 h o p}$ em função da taxa de transmissão de dados $(R)$ com o tamanho do pacote $N_{b}=100$ bits e com $n=10$ nós.

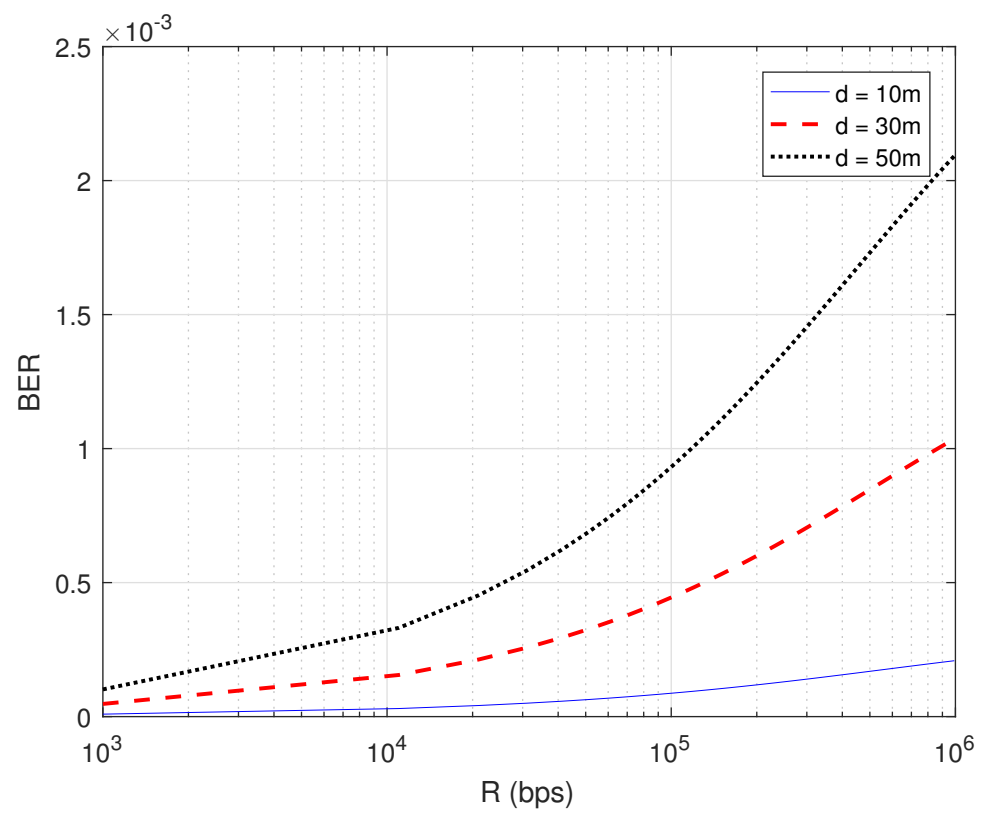

Figura 4. Taxa de erro de bit (Eq. (14)) em função da taxa de transmissão de dados $(R)$.

função do tamanho do pacote de dados (Eq. (20)). Observa-se que o consumo mínimo de energia se dá para $N_{b}$ entre 100 e 1000 bits, dependendo da distância entre os nós.

Comparando a Figura 6 para uma rede com $n=10$ nós e a Figura 7 para um cenário com apenas $n=2$ nós comunicantes, observa-se no cenário de dois nós um melhor desempenho, pois neste caso resulta menor probabilidade de erro na transmissão devido à ausência de interferência dos outros nós da rede e consequente menor consumo 


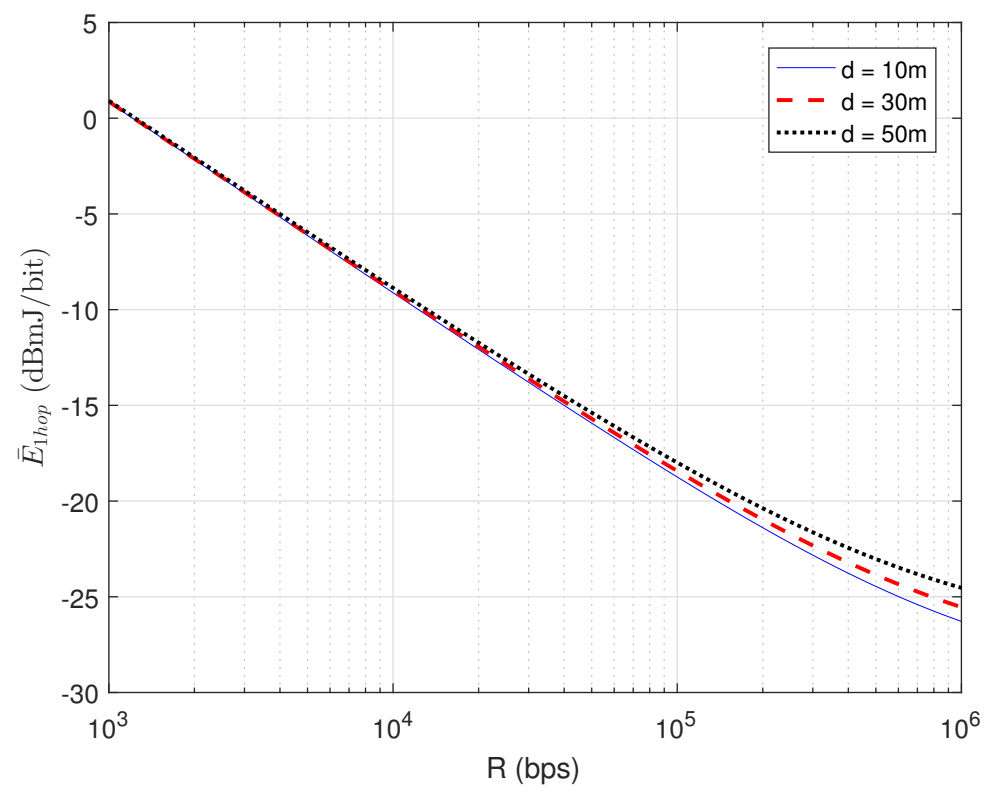

Figura 5. $\bar{E}_{1 h o p}$ em função da taxa de transmissão de dados $(R)$ com o tamanho do pacote $N_{b}=100$ bits e com $n=2$ nós comunicantes.

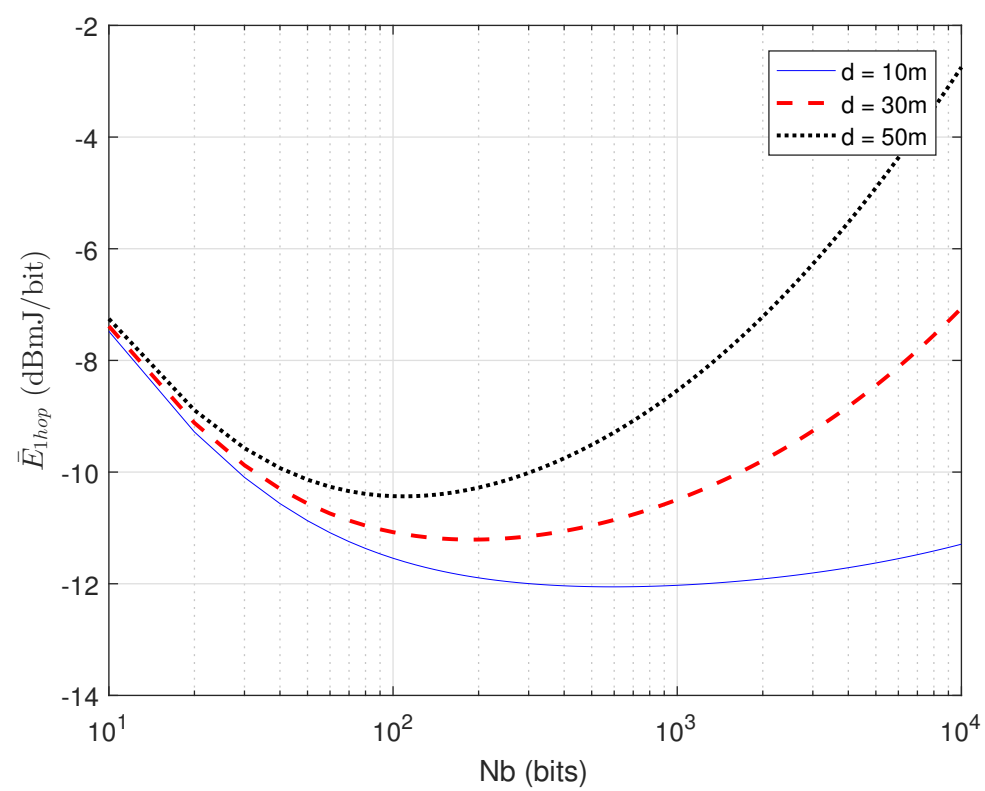

Figura 6. $\bar{E}_{1 h o p}$ em função do tamanho do pacote $\left(N_{b}\right)$ com uma taxa de transmissão $R=250$ kbps e $n=10$ nós.

de energia.

Por fim, para uma completa visualização da variação da energia consumida em um salto, a Figura 8 ilustra o comportamento como uma função simultânea da taxa de transmissão de dados e do tamanho do pacote. 


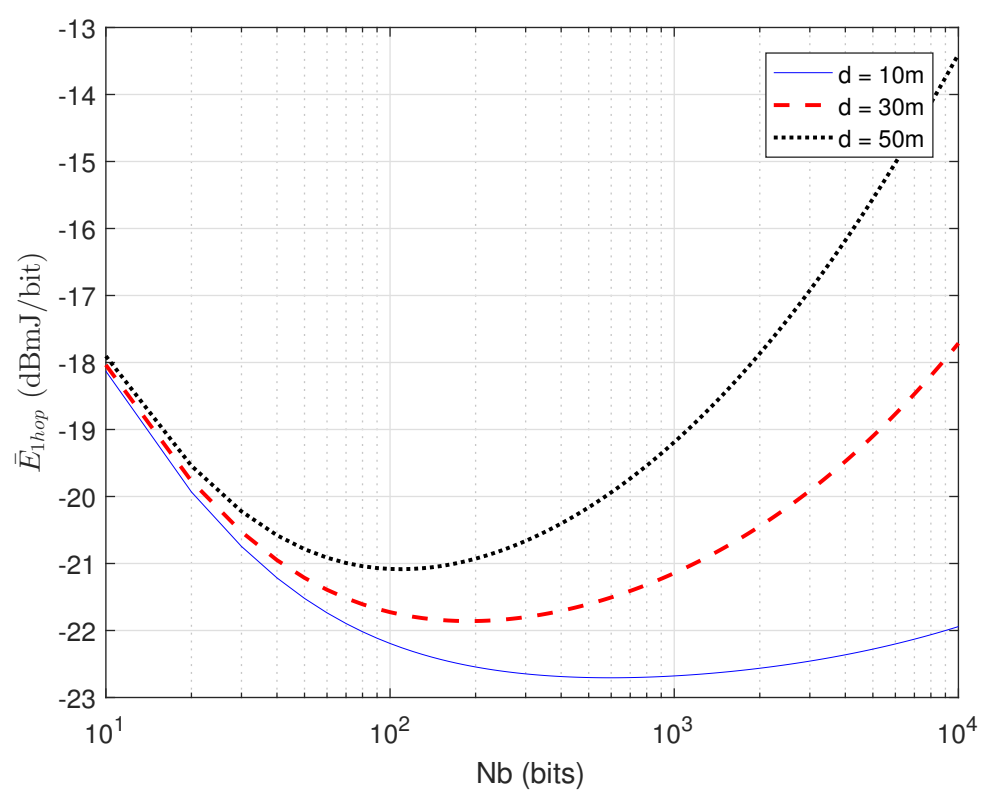

Figura 7. $\bar{E}_{1 h o p}$ em função do tamanho do pacote $\left(N_{b}\right)$ com uma taxa de transmissão $R=250 \mathbf{k b p s}$ e $n=2$ nós comunicantes.

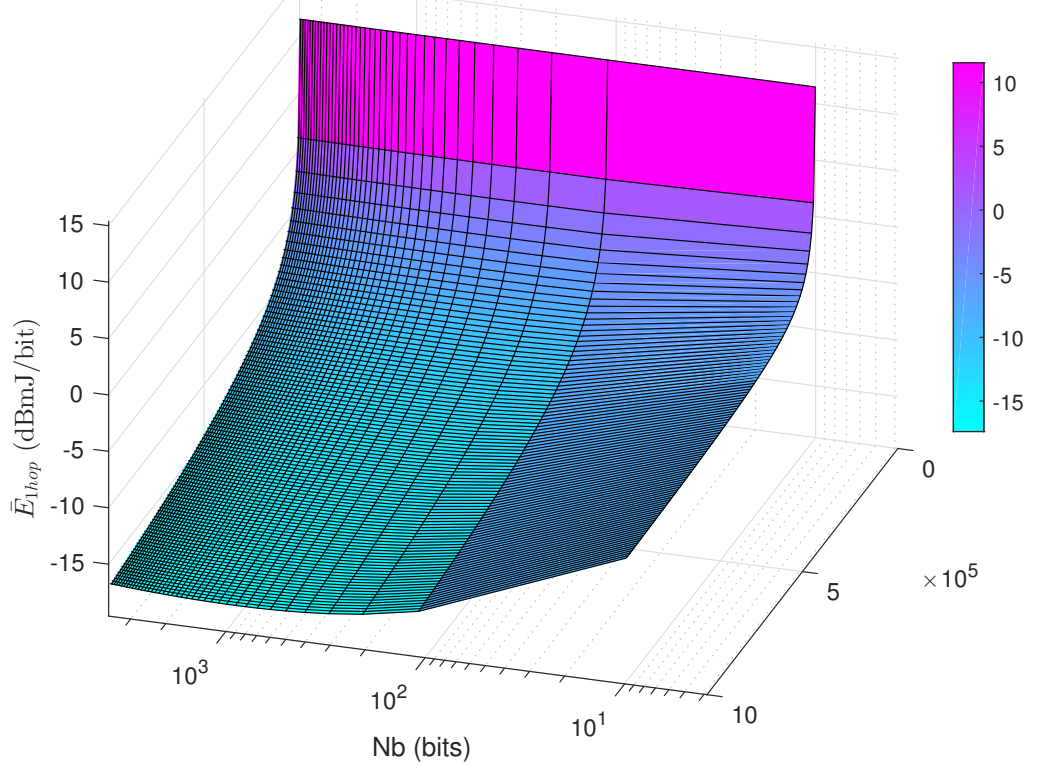

Figura 8. $\bar{E}_{1 h o p}$ em função da taxa de transmissão de dados $(R)$ e em função do tamanho do pacote $\left(N_{b}\right)$ para $n=10$ nós na rede e distância $d=10$ metros entre o par de nós comunicantes.

\section{Conclusões}

Este trabalho teve por finalidade investigar o comportamento do consumo médio de energia por bit para uma transmissão bem sucedida em uma rede ad hoc sem fio de um único salto. Uma das contribuições do estudo foi partir de uma robusta formulação de consumo 
de energia empregada na literatura e ampliá-la para considerar o protocolo de acesso ao meio Aloha. Outra contribuição do trabalho foi determinar a potência ótima de transmissão em função da taxa de transmissão de dados e do tamanho do pacote que resulte reduzido consumo de energia.

Como trabalhos futuros, pretende-se investigar o cenário de um salto e de múltiplos saltos de forma multi-objetiva integrado com o protocolo MAC. Procurar-seá ainda comparar e refinar o modelo em simuladores de redes, aplicando um protocolo MAC mais eficiente, e também testando em módulos (kits) práticos de sensores.

\section{Agradecimentos}

Este trabalho foi financiado em parte pela Fundação de Amparo a Ciência e Tecnologia do Estado de Pernambuco (FACEPE), pela Coordenação de Aperfeiçoamento de Pessoal de Nível Superior (CAPES) e pelo Conselho Nacional de Desenvolvimento Científico e Tecnológico (CNPq).

\section{Referências}

Abdulhadi, S., Naeem, M., Jaseemuddin, M., and Anpalagan, A. (Jun. 2013). Optimized packet size for energy efficient cooperative wireless ad-hoc networks. In IEEE International Conference on Communications Workshops (ICC), Budapest, Hungary.

Akbas, A., Yildiz, H. U., Tavli, B., and Uludag, S. (2016). Joint optimization of transmission power level and packet size for wsn lifetime maximization. IEEE Sensors Journal, 16(12):5084-5094.

Feeney, L. M. and Nilsson, M. (Apr. 2001). Investigating the energy consumption of a wireless network interface in an ad hoc networking environment. In Twentieth Annual Joint Conference of the IEEE Computer and Communications Societies (INFOCOM). Proceedings, volume 3, Anchorage, USA.

Gao, J. (2002). Analysis of energy consumption for ad hoc wireless sensor networks using a bit-meter-per-joule metric. IPN Progress Report, 42(150):1-19.

Goldsmith, A. (2005). Wireless communications. Cambridge university press.

Goratti, L., Yaprak, E., Savazzi, S., and Pomalaza-Raez, C. (2014). An urn occupancy approach for modeling the energy consumption of distributed beaconing. IEEE/ACM Transactions on Networking (TON), 22(1):203-216.

Gorce, J.-M., Zhang, R., and Parvery, H. (2007). Impact of radio link unreliability on the connectivity of wireless sensor networks. EURASIP Journal on Wireless Communications and Networking, 2007(1):1-16.

Gupta, A. L. and Shekokar, N. (Mar. 2016). A novel approach to improve network lifetime in wsn by energy efficient packet optimization. In IEEE International Conference on Engineering and Technology (ICETECH), Coimbatore, India.

Ito, A., Kato, T., Hatano, H., Sato, M., Watanabe, Y., Utsunomiya, E., Hiramatsu, Y., and Sato, F. (Oct. 2016). A study on designing ad hoc network protocol using bluetooth low energy. In 7th IEEE International Conference on Cognitive Infocommunications (CogInfoCom), Wroclaw, Poland. 
Karl, H. and Willig, A. (2007). Protocols and architectures for wireless sensor networks. John Wiley \& Sons.

Li, N., Lin, K., Yong, S., Chen, X., Wang, X., and Zhang, X. (Nov. 2015). Design and implementation of a mac protocol for a wearable monitoring system on human body. In IEEE 11th International Conference on ASIC (ASICON), Chengdu, China.

Liu, X., Xiong, N., Li, W., and Xie, Y. (2015). An optimization scheme of adaptive dynamic energy consumption based on joint network-channel coding in wireless sensor networks. IEEE Sensors Journal, 15(9):5158-5168.

Mobin, I., Mohammed, N., and Momen, S. (Feb. 2017). Optimal range estimation for energy efficient dynamic packet size. In International Conference on Electrical, Computer and Communication Engineering (ECCE), Cox's Bazar, Bangladesh.

Rana, K. and Zaveri, M. (2011). A-star algorithm for energy efficient routing in wireless sensor network. Trends in Network and Communications, pages 232-241.

Roberts, L. (1975). Aloha packet system with and without slots and capture. ACM SIGCOMM Computer Commun. Review, 5(2):28-42.

Sadiq, A. S., Almohammad, T. Z., Khadri, R. A. B. M., Ahmed, A. A., and Lloret, J. (May. 2017). An energy-efficient cross-layer approach for cloud wireless green communications. In Second International Conference on Fog and Mobile Edge Computing (FMEC), Valencia, Spain.

Shehab, A., Elhoseny, M., Sahlol, A. T., and El Aziz, M. A. (Mar. 2017). Self-organizing single-hop wireless sensor network using a genetic algorithm: Longer lifetimes and maximal throughputs. In IEEE International Conference on Intelligent Techniques in Control, Optimization and Signal Processing (INCOS), Srivilliputhur, India.

Stankovic, J. A. and He, T. (2012). Energy management in sensor networks. Phil. Trans. R. Soc. A, 370(1958):52-67.

Tang, Y.-J., Lee, C.-W., Lin, M.-H., Liu, B.-H., and Tsai, M.-J. (May. 2017). Energy consumption reduction methods of geographic routing protocols with out-of-date location information in mobile ad hoc networks. In IEEE International Conference on Communications (ICC), Paris, France.

Ye, W., Heidemann, J., and Estrin, D. (Jun. 2002). An energy-efficient mac protocol for wireless sensor networks. In Twenty-First Annual Joint Conference of the IEEE Computer and Communications Societies (INFOCOM). Proceedings, volume 3, New York, USA.

Zhang, R. and Gorce, J.-M. (Mar. 2008). Optimal transmission range for minimum energy consumption in wireless sensor networks. In Wireless Communications and Networking Conference (WCNC), Las Vegas, USA.

Zuniga, M. and Krishnamachari, B. (Oct. 2004). Analyzing the transitional region in low power wireless links. In First Annual IEEE Communications Society Conference on Sensor and Ad Hoc Communications and Networks (SECON), Santa Clara, USA. 